\title{
Effect of ultrasonic strain on p-type silicon wafers
}

\author{
Kazuki Tsuruta $^{1 \dagger}$, Masaki Mito ${ }^{1}$, Takuma Nagano ${ }^{1}$, Yuki Katamune ${ }^{2}$ and \\ Tsuyoshi Yoshitake ${ }^{2}\left({ }^{1}\right.$ Kyushu Inst. of Tech.; ${ }^{2}$ Kyushu Univ.)
}

\section{Introduction}

In the study of ferromagnets, the magnetization $M$ is an attractive physical parameter, and it has conjugate relation with the magnetic field $H$. On the other hand, the important quantity for ferroelectrics is the electric polarization $D$ that has conjugate relation with the electric field $E$. Thus, we manipulate attractive physical quantities by external fields that have conjugate relation with them.

However, in multiferroic materials accompanying ferromagnetic and ferroelectric properties, it has been known that $D$ can be manipulated by $H$ as well as $E$ [1], and $M$ can be done by $E$ as well as $H[2]$. Thus, this approach utilizing cross-correlation expands the possibility of material manipulation.

Abovementioned attractive condensed state originates from the many-body effects of electron on the lattice, and it should be manipulated by applying any stress to the crystal. Indeed there have been a lot of studies by using high-pressure experiments using pressure apparatus or strain experiments using piezoelectric substrates. However, the manipulation is stationary, and it is unfavorable for the intentional high-speed manipulation. Thus we are attempting the material manipulation by the use of dynamic stress with high frequency (here, we term it "ultrasonic strain"). Here, it is stressed that in the present study, the piezoelectric device is used as a source of time-dependent external field.

As the target, we paid much attention to semiconductors, and hoped the simplest semiconductor.

\section{Experimental method}

A target material is p-type silicon. We used a p-type Si wafer with the orientation along [110], and measured the electric resistance using two-terminal method. The surface size of the $\mathrm{Si}$ wafer is $2.5 \mathrm{~mm} \times 5 \mathrm{~mm}$, and the height is 0.13 $0.16 \mathrm{~mm}$. The electric resistivity of the wafer itself is $9-12 \mathrm{ohm} / \mathrm{cm}$, and the Pd electrode is deposited as electric contact.

As the source device of the ultrasonic strain, we used the commercial piezoelectric ceramics with the resonating frequency of about $1 \mathrm{MHz}$ whose the surface area is $6 \times 5 \mathrm{~mm}^{2}$. In the electric transport measurement as a function of temperature, the resonating frequency was confirmed at each measurement temperature. The $\mathrm{Si}$ wafer was mounted with silicon grease on the piezoelectric device as shown Fig. 2 (a). The piezoelectric device has the sufficient thermal contact with a copper plate in order to release the heat due to the resonation to the copper plate, i.e. the thermal bath. The ac voltage source was connected to piezoelectric device, and further the oscilloscope was connected to the circuit in the parallel style as shown Fig.2 (b). Furthermore, the dc current source and the digital voltmeter were connected to the sample as shown Fig.2 (b).

As the refrigerator covering from $5 \mathrm{~K}$ to 400 $\mathrm{K}$, a commercial SQUID (Superconducting Quantum Interference Device) magnetometer (Quantum Design Japan, Inc.) was used. The region enclosed in the dashed line in Fig.2 (b) corresponds to the sample cavity of the SQUID magnetometer, and the one-way distance from ac voltage source to the piezoelectric device is about $2 \mathrm{~m}$.

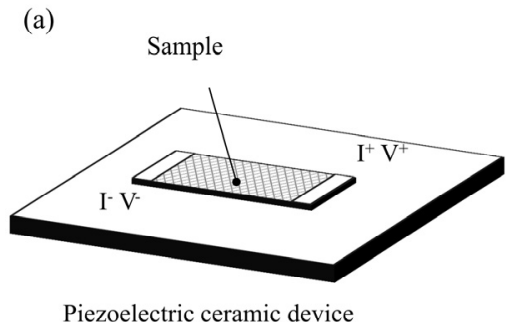

(b)

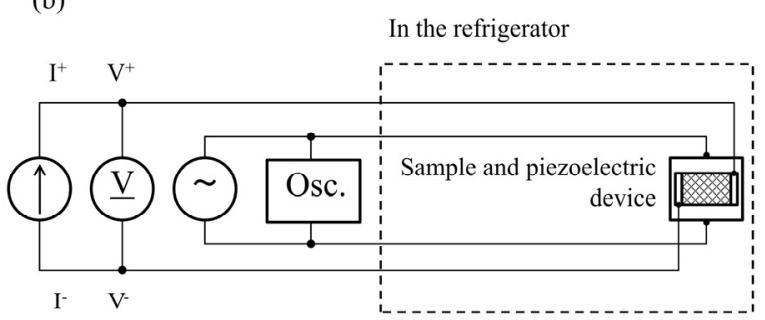

Fig.2 (a) Layout of the sample mounted on the piezoelectric device, (b) Overview of measurement system of the electric resistance. 


\section{Results}

Figure 3 shows the temperature dependencies of the electric resistance $(R)$ for each applied voltage $(V)$ in p-type Si wafer with the orientation along [110]. The number in the figure stands for the turn of each measurement. Without ultrasonic strain (described as "OFF" in the figure), the temperature region of more than $200 \mathrm{~K}$ corresponds to the saturation range. At around $200 \mathrm{~K}$, the border between the saturation range and impurity one, the electric resistance increases with increasing $V$. The change in the electric resistance is over $2000 \Omega$ that corresponds to more than quadruple of the initial resistance. As seen in "OFF 2nd" and "OFF 3rd",

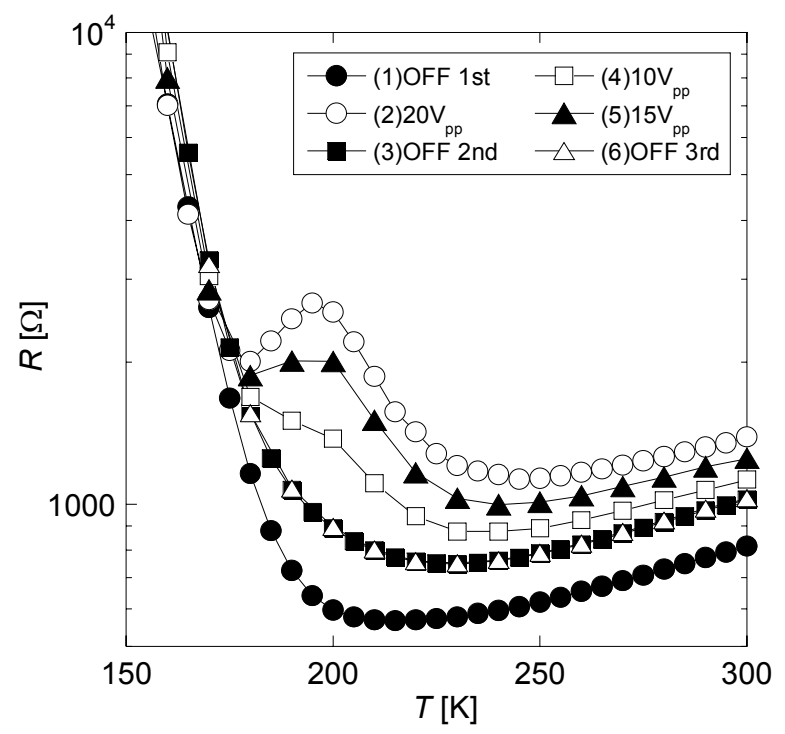

Fig.3 Temperature dependencies of the electrical resistance of p-type $\mathrm{Si}$ with the orientation along [110]

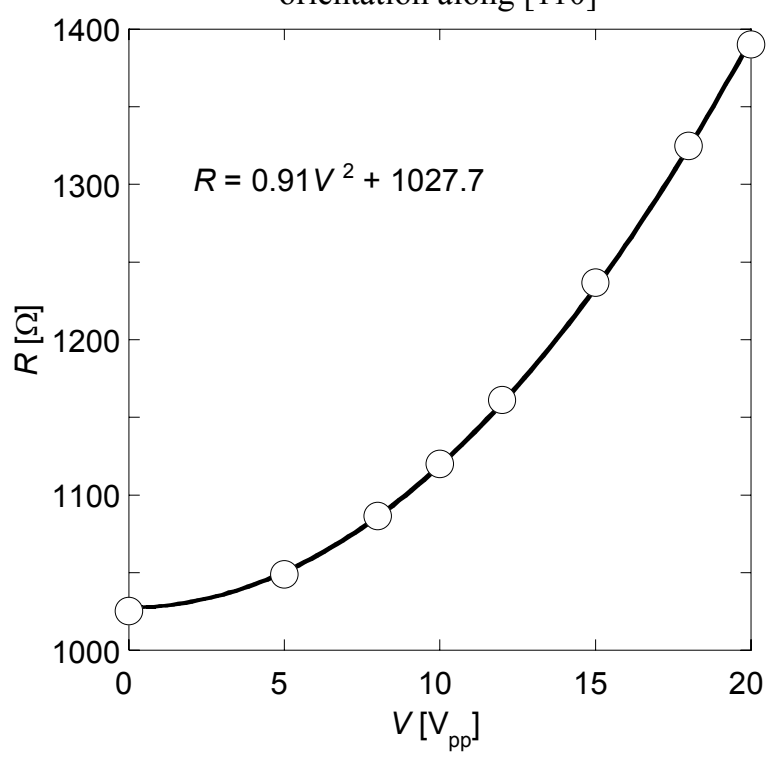

Fig.4 Electrical resistance as a function of voltage applied in p-type Si with the orientation along [110] when the $\mathrm{Si}$ wafer once experienced the strain corresponding to $20 \mathrm{~V}_{\mathrm{pp}}$, the electric resistance did not return to the initial one ("OFF 1st"), probably due to the residual dislocation.

Figure 4 shows the $V$ dependence of the electric resistance at $300 \mathrm{~K}$. The electric resistance increases in proportion to the square of $V$. This is attributed to the fact that the magnitude of ultrasonic strain is proportional to power consumed at piezoelectric device. We consider that the reduction of mobility and/or carrier number bring about the increase in the electric resistance. It has been reported that mobility of carrier is enhanced by squashing and elongating the $\mathrm{Si}$ wafer [3], exhibiting the inconsistent behavior with the present results. Finally, we stress that the time periodicity of manipulation can be controlled by adjusting on-and-off of the voltage applied to the piezoelectric device.

\section{Conclusion}

We have succeeded in manipulating the electric resistance by the use of the dynamic stress (the ultrasonic strain) on the semiconductor $\mathrm{p}$-Si. This approach could be developed in the scale of the real time manipulation of semiconductor. In order to elucidate the mechanism of artificial manipulation of electric resistance, we are planning the Hall effect measurement.

\section{Acknowledgment}

This work was supported by the Grand-in-Aid for Scientific Research on Innovative Areas, 'Bulk Nanostructured Metals' (KAKENHI 25102709).

\section{References}

1. S. Ishiwata, Y. Taguchi, H. Murakawa, Y. Onose and Y. Tokura: J. Phys. 150 (2009) 042073.

2. N. Hur, S. Park, P. A. Sharma, J. S. Ahn, S. Guha and S-W. Cheong: Nature 429 (2004) 392.

3. M. L. Lee and E. A. Fitzgerald: J. Appl. Phys. 97 (2005) 011101. 